\title{
REFLECTIONS \\ SATVI - after 10 years closing in on a new and better vaccine to prevent tuberculosis
}

\author{
Willem A Hanekom, Anthony Hawkridge, Hassan Mahomed, Thomas J Scriba, Michele Tameris, Jane Hughes, \\ Mark Hatherill, Cheryl L Day, Gregory D Hussey
}

The vision of the South African Tuberculosis Vaccine Initiative (SATVI) (http://www.satvi.uct.ac.za) is 'A World Without TB' and our mission is 'Innovative and high-quality TB vaccine research in Africa, to impact the global epidemic. Over the last 10 years, our focus has been two-fold: first, clinical trials of BCG and of new candidate vaccines, and second, complementary research that addresses critical questions in tuberculosis (TB) vaccine development. SATVI is now widely regarded as the leading TB vaccine clinical research site in the world.

S Afr Med J 2012;102(6):438-441
New, better vaccines: the answer to the global tuberculosis epidemic

In 2010 tuberculosis (TB) disease occurred in an estimated 8.8 million persons globally, causing 1.45 million deaths. ${ }^{1}$ South Africa has not been spared: approximately $1 \%$ of our population develops TB disease every year. ${ }^{1}$ About $60 \%$ of our TB patients also have HIV infection, placing us at the epicentre of the TB/HIV co-epidemic. ${ }^{1}$ While the global TB incidence has dropped, including on the continent of Africa, our country's disease rates have remained relatively static over the last few years. ${ }^{1}$

Advances in TB diagnosis and treatment are needed to control the epidemic. ${ }^{1,2}$ An effective vaccine would constitute the most sustainable intervention. ${ }^{1}$ The current TB vaccine, BCG, is important for prevention of severe forms of TB in infancy and early childhood, but affords variable efficacy - mostly poor - against lung TB. Adults with lung TB, particularly those who are not infected with HIV (Middelkoop et al., personal communication), spread the pathogen. Therefore new, better vaccination strategies should primarily aim to prevent disease in this population while including other populations at high risk of TB disease, such as infants/young children and HIVinfected persons.

\section{A brief history of SATVI}

SATVI was founded in 2001. Initially known as the 'BCG study unit', it was located within the Child Health Unit of the School of Child and Adolescent Medicine of the University of Cape Town's Faculty of Health Sciences. An immunology laboratory at Groote Schuur Hospital was later added to support core clinical trial activities.

A site for conducting clinical studies was established in the Boland Overberg region, about $110 \mathrm{~km}$ from Cape Town. The

All authors are current or previous lead investigators at the South African Tuberculosis Vaccine Initiative (SATVI), Faculty of Health Sciences, University of Cape Town. Professor Willem A Hanekom is the current Director of SATVI. Professor Gregory D Hussey was the founding Director, and Drs Anthony Hawkridge and Hassan Mahomed were previous Co-Directors. site's research activities cover an area of about $40000 \mathrm{~km}^{2}$, with a population of 350 000. Site selection has been critical for SATVI's success, the primary determinant being high reported rates of $\mathrm{TB}$, allowing end-points of vaccine trials to be achieved with reasonable sample sizes. Additionally, good transport, telecommunications and power infrastructure, availability of qualified or trainable staff and a relatively stable local population were essential, as also the well-functioning primary healthcare services, including adequate surveillance systems.

Operations at the field site are run from Brewelskloof Hospital in Worcester, a dedicated TB hospital for the region, and Worcester Hospital, a secondary level hospital. Two satellite site offices have been established, one in Robertson and one in Ceres.

The Cape Town operations of SATVI, including its state-of-theart immunology laboratory, are based at the Institute of Infectious Disease and Molecular Medicine at UCT's Faculty of Health Sciences, where extensive human resource and information technology and data management infrastructure exist.

The personnel complement of SATVI has grown from 3 to more than 190 persons over 10 years, more than 20000 participants have been enrolled into studies, funding support now exceeds R50 million a year, 80 research articles have been published in mid- to highimpact journals, and multiple $\mathrm{PhD}, \mathrm{MSc}$ and MPH candidates have graduated. SATVI leadership is represented on core international policy groups involved in TB vaccine development and in related, translational immunology.

\section{Clinical trials: BCG and new vaccines The 'BCG Trial'}

Our initial project was a phase IV randomised clinical trial intended to assess whether the route of BCG administration to newborns determined efficacy in protecting against childhood TB. The study enrolled 11680 infants at birth, over a period of 3.5 years, and showed equivalence in TB disease rates following BCG vaccination by an intradermal route and by a percutaneous route. ${ }^{3}$ This study was critical for developing infrastructure and capacity to conduct large-scale TB vaccine trials. The results may also guide policy in respect of BCG vaccination - it should be noted that the last clinical trial of a TB vaccine in infants was conducted by Rosenthal et al. in the 1930s and 1940s, long before the randomised placebo-controlled clinical trial came to be considered as the gold standard for evaluating vaccine interventions. ${ }^{4}$ 


\section{Trials of new TB vaccines}

More than $40 \mathrm{~TB}$ vaccine candidates are in preclinical development; 14 of these have entered human trials. ${ }^{5}$ New vaccines are likely to be administered within a heterologous prime boost strategy - using different vaccines to induce a first immune response and then boosting this immunity. The prime vaccine is likely to be a whole bacterium, which contains many antigens (which our immune systems recognise and react to), and can therefore induce broad immunity.

BCG is a classic prime vaccine. Novel prime vaccines in development include recombinant BCGs and even recombinant Mycobacterium tuberculosis (MTB). These viable organisms have been modified for greater safety, and to better induce an immune response important for protection against TB.

Current boost vaccines contain limited numbers of antigens, which are delivered either within a viral vector (vehicle) or together with specific adjuvants (see below). Viral-vectored boost vaccines use a modified cold virus, e.g. adenovirus-35, or a modified poxvirus such as modified vaccinia virus, to deliver antigens. Genetic modification of the viruses renders them replication-deficient and avirulent, and allows expression of the mycobacterial antigens to which the immune system reacts.

Subunit boost vaccines contain selected mycobacterial antigens, together with an adjuvant which delivers antigens in a stable formulation, stimulates the immune system for enhancing response to the antigen, and steers the immune response in the appropriate protective direction. The viruses of viral-vectored vaccines could also be viewed as adjuvants.

Most current vaccines contain antigens that are widely recognised by immune systems of persons latently infected or diseased with MTB, e.g. Ag85A, Ag85B and TB10.4. The dominance of the immune response to these antigens is thought to indicate a role in protection. It has recently been shown that some antigens may be preferentially expressed during latency, compared with early MTB infection or disease. Therefore, vaccine candidates developed to specifically target latency contain these co-called latency-associated antigens, e.g. Rv2660c in a vaccine termed H56. ${ }^{6}$

Vaccine testing typically involves phase I - IIa trials that focus on safety and immunogenicity, first in small groups of healthy adults, followed by studies in younger persons and in those with latent TB infection and/or HIV infection. Thereafter, phase IIb and III trials focus on efficacy to prevent TB disease, and involve much larger groups of participants.

SATVI has conducted clinical trials of 5 new TB vaccine candidates, ${ }^{6-8}$ in 11 different protocols. In early trials, 2 viral-vectored vaccines, MVA85A and Aeras-402, as well as adjuvanted subunit vaccines, $\mathrm{H} 4$ and $\mathrm{M} 72$, were shown to be safe and immunogenic in various populations. ${ }^{78} \mathrm{~A}$ current trial of $\mathrm{H} 56$ is the first in humans. Because we have experience of testing multiple vaccine candidates, we have been able to observe unique patterns of adverse events and of immune induction, depending on the candidate vaccine. These comparative data are important for deciding which candidates should enter later-phase clinical trials.

We are currently conducting early efficacy trials (phase IIb) of 2 candidates, MVA85A and Aeras-402, in infants. The results of the trial of MVA85A, involving 2797 infants, and conducted exclusively at SATVI, are expected to be available at the beginning of 2013.

\section{Complementary research: critical questions in TB vaccine development}

As a principle, SATVI uses registration-standard vaccine trials to explore other critical areas in vaccine development. These include, for example, novel approaches to informed consent, ${ }^{9}$ tools to measure the tuberculin skin test, ${ }^{10}$ approaches to diagnosing $\mathrm{TB}$ disease in children for late-phase vaccine trials ${ }^{11}$ and detailed studies of the vaccination-induced immune response., ${ }^{7,12}$ In addition, specific clinical studies have been conducted to address additional questions (highlighted below).

\section{Studies to define the TB epidemic}

Infants and adolescents are potential target groups for novel TB vaccines. Successful conduct of late-phase trials in these populations requires reliable data about the TB epidemic at a target site. Since data from public health structures are often suboptimal, we have completed 2 large-scale epidemiological studies.

A longitudinal study involving 4786 neonates showed that the incidence in children under 2 years of age was 1.5/100 person-years. We also wished to address whether active or passive follow-up is required for case-finding in efficacy trials: we showed that after 2 years of follow-up, quarterly home visits, combined with communitywide surveillance, detected significantly more cases, and at younger ages, compared with surveillance with only a single visit at the end of the study. ${ }^{14}$

The 'Adolescent cohort study' involved 6363 adolescents, who were followed up for 2 years. We showed an incidence rate of 0.45 (95\% CI 0.30 - 0.69) per 100 person-years. Black or coloured race, male gender, older age, household TB contact, low income and low education level were significant predictive factors for TB infection. In contrast to findings in infants, active surveillance did not significantly improve case detection. ${ }^{15}$

\section{Immunity induced by BCG in HIV-infected infants}

Our early studies focused on practical and rigorous methods for conducting sophisticated human immunology studies in rural field settings. ${ }^{16}$ These methods were further optimised for paediatric applications, such as studies aimed at gaining a better understanding of how our immune system interacts with MTB and BCG. We also applied this technology to evaluate immunogenicity of BCG in HIV-infected children: at the time that this study ${ }^{17}$ commenced, early antiretroviral therapy was not available for this population, and reports of complications from the vaccine (BCGosis) emerged. We showed that BCG induces very poor immunity in HIV-infected infants, both quantitatively and qualitatively, ${ }^{17}$ and have contributed to rational international policy regarding the use of BCG vaccination in HIV-infected infants.

\section{Biomarker studies}

SATVI's flagship immunology projects address the holy grail of vaccine development - immune correlates of protection against TB. Identification of such correlates would accelerate the screening of new, better vaccine candidates. Identification of prospective correlates of risk of TB disease would also accelerate vaccine development, by allowing smaller sample sizes in expensive late-phase trials, through targeted enrolment.

We used the 'BCG Trial', mentioned above, to determine correlates of risk of TB disease, following newborn vaccination with BCG. Host responses in blood stored from 10 weeks after newborn BCG vaccination were compared in infants who ultimately developed $\mathrm{TB}$ disease, and those who did not. As we know that T-cell immunity is important for protection, our first focus was on these responses. Remarkably, we have shown that this immunity, the most commonly measured in trials of new $\mathrm{TB}$ vaccines, did not correlate with risk of TB disease. ${ }^{18}$ Follow-up studies are focusing on unbiased 
approaches, such as whole-genome expression analysis, to identify novel correlates of risk of TB disease.

Cutting-edge unbiased analyses, such as RNA sequencing, are currently being used to determine correlates of risk of TB disease in samples from the 'Adolescent cohort study'. In a similar casecontrol design, blood products stored at 6-monthly intervals from adolescents who have developed TB disease, and those who have not, are being examined.

A definitive study of correlates of protection against TB disease is ultimately possible from our randomised controlled phase IIb trials, once efficacy has been shown.

\section{The performance of the QuantiFERON test in adolescents} One of the aims of the 'Adolescent cohort study' was to evaluate the performance of the QuantiFERON Gold-In Tube test (QFT) in a TB-endemic setting. This interferon- $\gamma$ release assay was developed as a replacement for the tuberculin skin test (TST), more commonly known as the 'Mantoux test'; both tests indicate the presence of latent MTB infection. Unlike the TST, the QFT requires a single visit only and is not affected by BCG vaccination or boosted by repeated testing. However, the QFT is more costly and requires some laboratory infrastructure. We completed more than 20000 longitudinal QFT tests in the 'Adolescent cohort study'. We learned about variation in the QFT, and found good agreement between the QFT and TST, both of which proved good predictors of the onset of TB disease. ${ }^{19}$ Importantly, we showed that the TST does not need to be replaced by the QFT in a high-burden setting such as South Africa.

\section{Alignment with key stakeholders Academic collaborations}

A major contributor to SATVI's success has been productive partnerships with relevant local and international academics. Our contribution has focused on what South Africa can do, and does extremely well - enrolling relevant participant populations, conducting high-quality clinical research, and bridging the gap from the participant to the laboratory bench. We have worked with partners to complement these areas in choosing, for example, not to set up high-throughput gene expression analysis locally, instead deferring that task to well-resourced laboratories overseas.

An example of a very successful partnership is the TB Vaccine Site Network (TBVACSIN), spearheaded by SATVI academics, with support from the Aeras Foundation and the European Developing Countries Clinical Trials Partnership (EDCTP). This collaboration involves a number of African sites, which work towards optimal and harmonised TB vaccine trial capacity. Aspects that are addressed include training, good clinical and laboratory practice, quality management, clinical and microbiological diagnosis of $\mathrm{TB}$, and vaccine trial immunology.

Two other consortia, involving a total of 16 African and Northern research groups and funded by the Gates Foundation, under its Challenges in Global Health umbrella, have been examples of successful collaborations to address specific scientific questions. The aim of the first has been to develop a post-infection vaccine: the developed vaccine, H56, is currently in a clinical trial at SATVI. The other has aimed to develop biomarkers of TB - all samples have been collected, and plans for analysis are underway.

\section{Community and health authorities}

Successful site development emphasises the importance of community engagement and community involvement. A community advisory board has served as a voice for the local community, guiding us in our research initiatives. We have also learned about the importance of community outreach events, and optimal communications through local media. Multiple benefits for the relatively socio-economically compromised community have emerged from the site's operations, including facilitated and improved healthcare, and a decreased incidence of severe forms of TB in infancy.

Political and practical support from the Departments of Health, Science and Technology, Education, and Social Development has also been critical for success. Involvement on all levels is important - from national ministers to national, provincial and district health departments. SATVI has had a strong social commitment to the local authorities, by providing TB-specific training of health professionals and infrastructure development, and by donating equipment to health facilities and schools. In turn, local authorities and schools have facilitated communication with participants about study endpoints (e.g. TB disease) reached by our study participants, and have donated space for our study procedures. We have shown that it is possible to design protocols and conduct projects that are mutually beneficial for investigators and service providers (and, indirectly, for sponsors).

\section{Our most valuable resource - our personnel}

Our research teams now include experts in clinical trial science, epidemiology, clinical aspects and immunology of $\mathrm{TB}$ and of vaccines, laboratory technology, training, quality assurance and quality control, regulation, information technology, data management, finance, administration and logistics.

Multiple postgraduate students are training within SATVI. In addition to formal academic learning, non-traditional training at our site was needed, as persons with specialised skills are in relative short supply. SATVI, in collaboration with the Aeras Foundation, developed a Professional Development Programme (PDP) to address these needs. As a result, each personnel member's training has been tailored to complement his/her brought experience, while addressing standards mandated by our research. The PDP has since been shared with other TB vaccine sites in Africa and India. Continued investment in our personnel has demanded that we change our approaches as new needs arise, or for efficiency. Following the PDP, our emphasis is on project-specific training, managed by project co-ordinators. We are also developing a formal human culture and capital plan.

\section{Optimal strategy/business structures}

As is the common experience of companies during rapid growth, SATVI has had to add structures on a relatively ad hoc basis from 2001 to 2011, to meet ever-increasing demands with the risk, however, of a loss of efficiency. In 2011, therefore, we conducted an academic and a strategic review, as a result of which SATVI's new strategy is based on sustainability, needs in the research environment, and a careful assessment of market forces. A new vision and mission has been devised, along with strategic objectives and a business plan, for the years ahead. This process will mark the transition of SATVI from 'adolescence' to 'adulthood'.

\section{Conclusion}

We are far from winning the war against TB. The emergence of extensively (and totally) drug-resistant MTB strains has highlighted the urgency for the development of better vaccines able to prevent all forms of the disease. A decade or two ago, prospects for a new TB vaccine were dim because many basic questions remain unanswered, but the last 10 years have seen rapid development. SATVI has made 
major contributions to TB vaccine development, and is committed to continue to work to achieve 'A World Without TB'.

Acknowledgements. The authors acknowledge contributions of every SATVI member, from 2001 to the present: our achievements would not have been possible without their superb commitment. We acknowledge also the participants who have volunteered for projects. Finally, we thank funders and sponsors, especially the Sequella Foundation and the Aeras Foundation, whose support enabled establishment of most of our infrastructure, the NIH, the Gates Foundation, EDCTP, the EU, the Wellcome Trust, the Elizabeth Glaser Paediatric AIDS Foundation, and the FDA.

1. World Health Organization. Global tuberculosis control (WHO Report 2010). http://www.who.int/tb/ publications/global_report/en/ (accessed January 2012).

2. Abu-Raddad L Sabatelli L Achterberg JT et al. Epidemiological benefits of more-effective tuberculosis vaccines, drugs, and diagnostics. Proc Natl Acad Sci USA 2009;106:13980-13985.

3. Hawkridge A, Hatherill M, Little F, et al. Efficacy of percutaneous versus intradermal BCG in the prevention of tuberculosis in South African infants: randomised trial. Br Med J 2008;337:a2052.

4. Rosenthal SR, Loewinsohn E, Graham ML, Liveright D, Thorne G, Johnson V. BCG vaccination against tuberculosis in Chicago. A twenty-year study statistically analyzed. Pediatrics 1961;28:622-641

5. Tuberculosis Vaccine Pipeline - 2011. Stop TB Working Group on New Vaccines. http://www.stoptb. org/wg/new_vaccines/assets/documents/TB\%20Vaccine\%20Pipeline $\% 2010 \% 20-\% 2003 \% 2021 \% 2011$. pdf; 2011 (accessed January 2012)

6. Aagaard C, Hoang T, Dietrich J, et al. A multistage tuberculosis vaccine that confers efficient protection before and after exposure. Nat Med 2011;17:189-194

7. Bel B, Tameris M, Mansoor N, et al. The novel tuberculosis vaccine, AERAS-402, induces robust and polyfunctional CD4+ and CD8+ T cells in adults. Am J Respir Crit Care Med 2010;181:1407-1417.

8. Hawkridge T, Scriba TJ, Gelderbloem S, et al. Safety and immunogenicity of a new tuberculosis vaccine, MVA85A, in healthy adults in South Africa. J Infect Dis 2008;198:544-552.

9. Mahomed H, Shea J, Kafaar F, Hawkridge T, Hanekom WA, Hussey GD. Are adolescents ready for tuberculosis vaccine trials? Vaccine 2008;26:4725-4730.

10. Geldenhuys $\mathrm{H}$, Verver $\mathrm{S}$, Surtie $\mathrm{S}$, et al. The tuberculin skin test: a comparison of ruler and calliper readings. Int J Tuberc Lung Dis 2010;14:1266-1271.

11. Hatherill M, Verver S, Mahomed H. Consensus Statement on Diagnostic End Points for Infant Tuberculosis Vaccine Trials. Clin Infect Dis 2012;54:493-501.

12. Scriba TJ, Tameris M, Mansoor N, et al. Modified vaccinia Ankara-expressing Ag85A, a novel tuberculosis vaccine, is safe in adolescents and children, and induces polyfunctional CD4+ T cells. Eur J Immunol 2010;40:279-290.

13. Scriba TJ, Tameris M, Mansoor N, et al. Dose-finding study of the novel tuberculosis vaccine, MVA85A, in healthy BCG-vaccinated infants. J Infect Dis 2011;203:1832-1843.

14. Moyo S, Verver S, Hawkridge A, et al. Tuberculosis case finding for vaccine trials in young children in high-incidence settings: a randomised trial. Int J Tuberc Lung Dis 2012;16:185-191.

15. Mahomed H, Hawkridge T, Verver S, et al. Predictive factors for latent tuberculosis infection among adolescents in a high-burden area in South Africa. Int J Tuberc Lung Dis 2011;15:331-336.

16. Hanekom WA, Hughes J, Mavinkurve M, et al. Novel application of a whole blood intracellular cytokine detection assay to quantitate specific T-cell frequency in field studies. J Immunol Methods 2004;291:185-195.

17. Mansoor N, Scriba TJ, de Kock M, et al. HIV-1 infection in infants severely impairs the immune response induced by Bacille Calmette-Guerin vaccine. J Infect Dis 2009:199-982-990.

18. Kagina BM, Abel B, Scriba TJ, et al. Specific T cell frequency and cytokine expression profile do not correlate with protection against tuberculosis, following BCG vaccination of newborns. Am J Respir Crit Care Med 2010:182:1073-1079.

19. Mahomed H, Hawkridge T, Verver S, et al. The tuberculin skin test versus QuantiFERON TB Gold(R) in predicting tuberculosis disease in an adolescent cohort study in South Africa. PLoS One 2011;6:e17984.

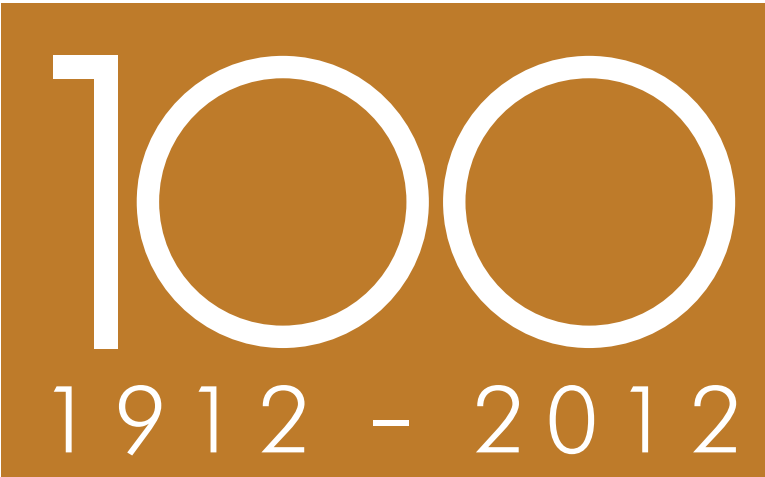

CELEBRATING THE PRESENT

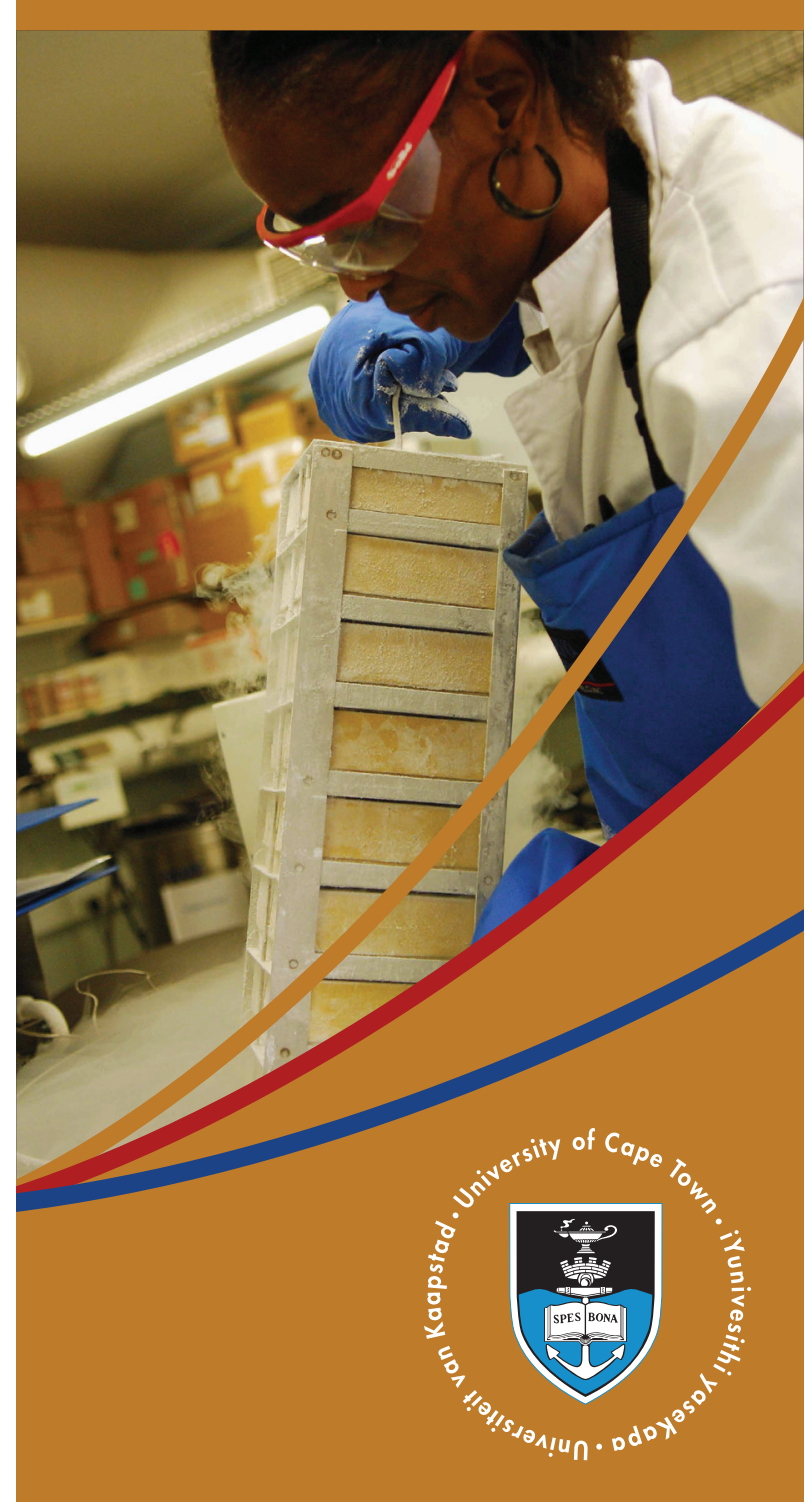

FACULTY OF HEALTH SCIENCES CENTENARY 